\title{
HPLC Separation of Phenolic Acids on Silica Gels Layer-by-Layer Modified with 6,10-Ionene and Dextran Sulfate
}

\author{
A. N. Ioutsi i \\ Scientific Centre for Expert Evaluation of Medicinal Products (SCEEMP) of the Ministry of Health of Russia, \\ Moscow 127051, Russia \\ Correspondence should be addressed to A. N. Ioutsi; freund-for-me@mail.ru
}

Received 3 August 2020; Revised 30 September 2020; Accepted 8 October 2020; Published 24 October 2020

Academic Editor: Victor David

Copyright (C) 2020 A. N. Ioutsi. This is an open access article distributed under the Creative Commons Attribution License, which permits unrestricted use, distribution, and reproduction in any medium, provided the original work is properly cited.

Using phenolic acids as an example, we continue to study the nature of the previously obtained multilayer sorbents for HPLC-silica gels modified up to two times with 6,10-ionene and dextran sulfate (DS). The chromatographic behavior of this class of compounds on the obtained sorbents was studied. A six-component mixture of sorbic, vanillic, sinapic, $p$-coumaric, caffeic, and ferulic acids was separated on the silica gel twice modified with 6,10-ionene and dextran sulfate in 15 min with a selectivity of up to 1.88 and a column efficiency of up to 26000 theoretical plates per meter. The possibility of separating the two classes of compounds-phenolic acids and basic nitrogen-containing pharmaceuticals - in a single mixture on silica gel, twice modified with 6,10-ionene and dextran sulfate, is shown.

\section{Introduction}

HPLC is an advanced form of liquid chromatography used in separating the complex mixture of molecules encountered in chemical and biological systems, in order to recognize better the role of individual molecules. During the survey of the literature, it was observed that, among the chromatographic techniques, HPLC has been the most widely used system.

HPLC provides a major service in answering many questions posed by the pharmaceutical industry $[1,2]$ and food industry [3-5]. HPLC is considered as one of the most important techniques of the last decade of the $20^{\text {th }}$ century. It became the method of choice for analytical support in many stages of quality control and assurance within the pharmaceutical industry. Recently, HPLC-MS has been used for assay of drugs. In addition to its application in analyzing the drugs, HPLC alone and with hyphenated technique has been applied to analyze the impurities of the pharmaceuticals and degradation products [6].

Pharmacology and the food industry are relevant and developing areas of industry today. In pharmacology and other industries, there has always been a need to use compounds that can increase the shelf life of products, maintain the properties of the main active ingredients in specific dosage forms, or sometimes just add interesting taste in the case of the children's medicines. Phenolic acids (PAs), a class of polar compounds, often act as sweeteners and preservatives. The requirements for the content of excipients, including PA, in medicines, are gradually being tightened in the standards of the European Directorate for the Quality of Medicines and Healthcare (EDQM), Guidelines of the International Council for Harmonization of Technical Requirements for Pharmaceuticals for Human Use (ICH). Therefore, monitoring their content is an important and urgent task. Evaluation of the content of preservatives and sweeteners in pharmaceuticals and other substances must be carried out directly at the factory $[7,8]$.

This problem is successfully being solved by HPLC on the mix-mode stationary phases, where both hydrophobic and hydrophilic sorbate-sorbent interactions are realized. Silica gels modified by various cationic and anionic polymers [9-13], including polysaccharides [14, 15], can serve as an example of such sorbents. Similar sorbents, based on polyelectrolyte layers of surface modifiers, have also been studied in capillary electrophoresis (CE) $[16,17]$. A fairly 
simple method of surface modification-layer-by-layer deposition of polyelectrolytes, including polysaccharides-has been used for several decades to create materials for separation methods. It was established that the deposition of polysaccharides carrying a negative charge on the inner surface of a quartz capillary led to an increase in the selectivity of separation of polar organic compounds by the CE method, as well as to an improvement in the reproducibility of the results [18].

In previous works, we obtained quartz capillaries and silica gels modified with 6,10-ionene and DS [18, 19]. An initial assessment of the surface properties of such polyelectrolyte sorbents for HPLC was carried out using nitrogencontaining compounds as an example. It was established that the surfaces of such sorbents have both hydrophobic and hydrophilic as well as ion-exchange properties (due to the presence of hydrophobic anions and cations on the surface). Such polyelectrolyte sorbents make it possible to successfully separate polar organic substances of a basic nature.

In this work, we focused on gaining a deeper insight into the surface properties of our sorbents while broadening the range of their potential applications by studying the separation of a fundamentally different class of compounds-phenolic acids. We have also investigated the possibility of the simultaneous separation of PA and nitrogen-containing pharmacologically active compounds. Benzoic, nicotinic, gallic, salicylic, ferulic, sinapic, $p$-coumaric, syringic, caffeic, and vanillic acids were used as model compounds (Figure 1). Sorbic acid was also included in the study to assess the effect of a benzene ring fragment on retention. Atenolol, nadolol, chlorpheniramine, carbinoxamine, and tetrahydrozoline were included in the test mixture of the two compound classes as nitrogen-containing basic compounds.

\section{Experimental}

2.1. Materials and Reagents. All chemicals were of analytical grade, and all solvents were of HPLC grade. Analytical standards for sorbic, benzoic, nicotinic, gallic, salicylic, ferulic, sinapic, $p$-coumaric, syringic, caffeic, and vanillic acids were purchased from Sigma-Aldrich (USA). Analytical standards for atenolol, nadolol, chlorpheniramine, carbinoxamine, and tetrahydrozoline were also obtained from Sigma-Aldrich (USA). Sodium citrate, sodium hydroxide, potassium phosphate trihydrate, and dipotassium phosphate were obtained from Reachim (Russia). Dextran sulfate was purchased from Sigma-Aldrich (USA). Kromasil 100-5-Sil silica gel (particle size $5 \mu \mathrm{m}$, surface area $300 \mathrm{~m}^{2} / \mathrm{g}$, pore size $100 \AA$, Eka Chemicals (Sweden)) was used as matrices for the synthesis of sorbents. 6,10-Ionene was synthesized in-house using the Menshutkin reaction [20].

Mobile phases (MPs) were prepared from acetonitrile ("PanReac," Spain), methanol ("Honeywell," Germany), isopropyl alcohol (LiChrosolv, Germany), and tetrahydrofuran (THF) ("Reachim," Russia). Buffers were prepared in deionized water under $\mathrm{pH}$ control with the S20 SevenEasy $\mathrm{pH}$ meter (Mettler Toledo, Switzerland).
2.2. Instrumentation. The HPLC system consists of LC-20 Prominence liquid chromatograph (Shimadzu, Japan) equipped with an autosampler and SPD-M20 A diode array detector (Shimadzu, Japan). The volume of the injection loop was $20 \mu \mathrm{l}$. Mobile phases were filtered through a $0.45 \mu \mathrm{m}$ membrane filter (Millipore, Ireland) and degassed with an US degasser.

Data collection and chromatogram processing were performed using LC Solution software (Shimadzu). The flow rate of the MP was $0.7-1.0 \mathrm{ml} / \mathrm{min}$. Detection was carried out at $\lambda=220,235$ (salicylic acid) and 220 (benzoic acid) $\mathrm{nm}$. The steel columns $100 \times 4.6 \mathrm{~mm}$ and $150 \times 4.6 \mathrm{~mm}$ in size were filled with the synthesized sorbents using a Knauer K-1900 pump (Knauer, Germany) under 350 bar pressure using the suspension method. For this, a weighed portion of $1.5 \mathrm{~g}$ of the sorbent was added to $20 \mathrm{ml}$ of the solvent, and the resulting suspension was homogenized in an ultrasonic bath for 2 min prior to filling the column.

2.3. Preparation of Stock Solutions. Stock solutions of acids with a concentration of $100 \mu \mathrm{g} / \mathrm{ml}$ were prepared by dissolving an exact weight of the analytical standard in deionized water. All solutions were stored at $4^{\circ} \mathrm{C}$.

2.4. 6,10-Ionene Synthesis. Equimolar solutions of $N, N, N^{\prime}, N^{\prime}$ tetramethyl-1,6-hexanediamine and 1,10-dibromodecane were dissolved in DMF to obtain a $1 \mathrm{M}$ solution for each of the components. The mixture was stirred on a magnetic stirrer for 24 hours at room temperature. During the reaction, precipitation of the resulting polymer was observed. The end of the reaction was monitored visually until the cessation of the increase in sediment volume. The reaction mixture was poured with stirring into an approximately 20 -fold volume of anhydrous acetone. Then, the precipitate was filtered on a waterjet pump and washed with two $10 \mathrm{ml}$ portions of acetone. The polymer was dried under vacuum generated by an oil pump $\left(2 \cdot 10^{-2} \mathrm{~mm} \cdot \mathrm{Hg}\right)$ for 4 hours.

2.5. Layer-by-Layer Modification Protocol. Kromasil 100-5Sil silica gel was activated with a $0.001 \mathrm{M} \mathrm{NaOH}$ solution and then washed 2 times with deionized water. $2.8 \mathrm{~g}$ of activated silica gel was treated with a water solution $(0.07 \mathrm{mg} / \mathrm{ml})$ of 6,10 -ionene for 20 minutes at $60^{\circ} \mathrm{C}$ in an ultrasonic bath. The solution was left for 24 hours and then washed twice with deionized water. The resulting sorbent was treated with a DS solution $(12.5 \mathrm{mg} / \mathrm{ml})$, while stirring on a mechanical shaker for 40 minutes. The resulting mixture was left overnight. The next day, the resulting stationary phase 1 (SP-1) was washed with deionized water. To obtain twice modified silica gel-SP2 -the described procedure was repeated with SP-1. First studies of the composition of the obtained sorbents and reagents are described in [19]. General structure of the synthesized stationary phases is given in Figure 2. 
<smiles>O=C(O)c1ccccc1</smiles>

Benzoic acid<smiles>O=C(O)c1cccnc1</smiles>

Nicotinic acid<smiles>O=C(O)c1ccccc1O</smiles>

Salicylic acid<smiles>O=C(O)/C=C/c1ccc(O)c(O)c1</smiles>

Caffeic acid<smiles>COc1cc(C(=O)O)cc(OC)c1O</smiles>

Syringic acid<smiles>O=C(O)/C=C/c1ccc(O)cc1</smiles>

Coumaric acid<smiles>C/C=C/C=C/C(=O)O</smiles>

Sorbic acid<smiles>COc1cc(/C=C/C(=O)O)ccc1O</smiles>

Ferulic acid<smiles>COc1cc(C(=O)O)ccc1O</smiles>

Vanillic acid<smiles>O=C(O)c1cc(O)c(O)c(O)c1</smiles>

Gallic acid

Figure 1: Chemical structures of phenolic acids and sorbic acid.

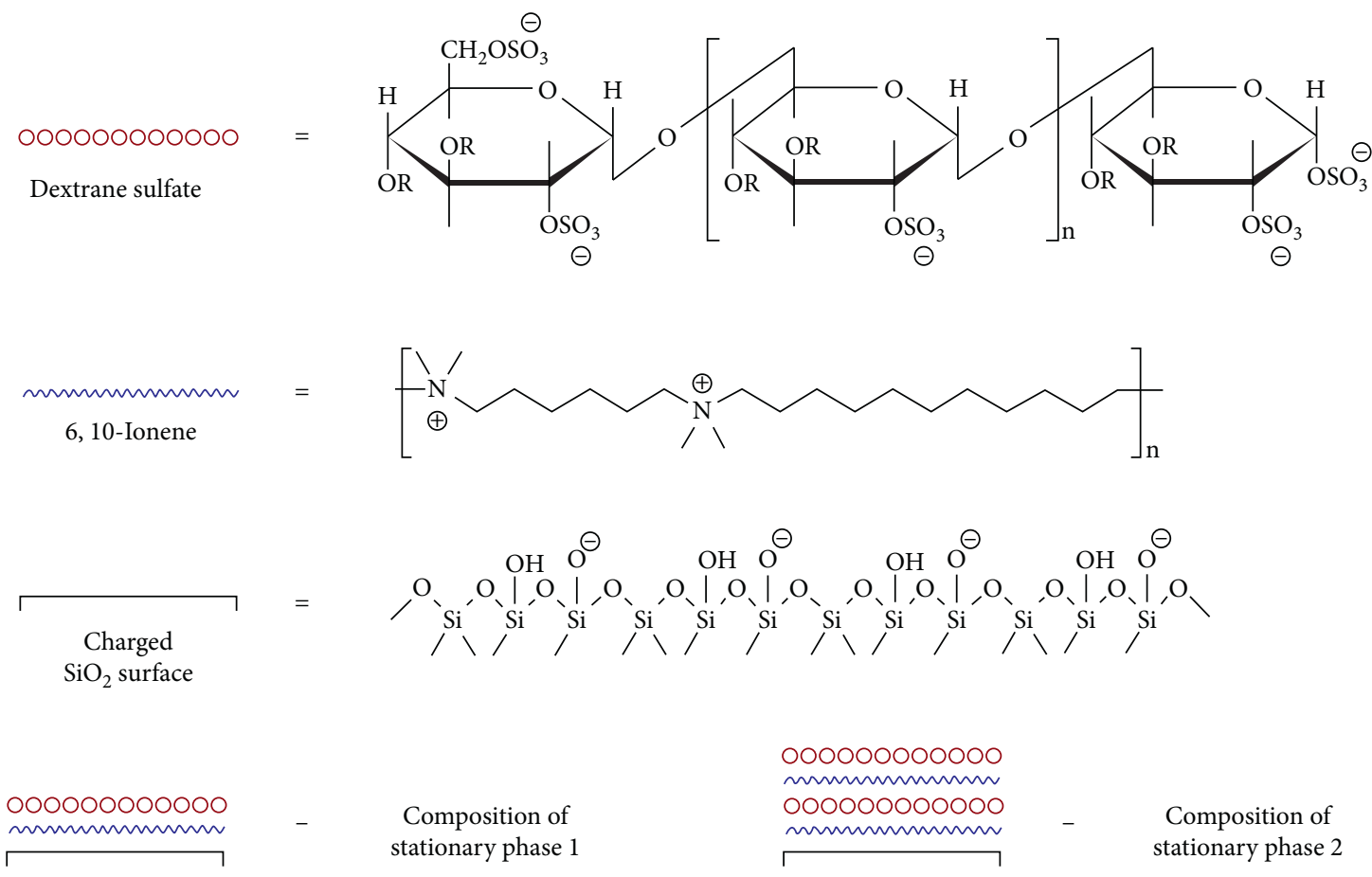

Figure 2: General structures of SP-1 and SP-2.

2.6. Coating Stability Evaluation. For the studied compounds (phenolic acids and nitrogen-containing compounds) at the evaluated conditions (methanol/buffer, acetonitrile/buffer mixtures, $\mathrm{pH}$ in the $4.8-7.6$ range), no significant deviations in retention times and peak spreading were observed for 9 months, including our work [21] with mass spectrometric detection. After that, the retention times of the investigated compounds changed by $0.5-2.5 \%$.

\section{Results and Discussion}

\subsection{Method Development}

3.1.1. Silica Gel Modified with 6,10-Ionene and Dextran Sulfate (SP-1). The elution order of acids from the surface of SP-1 was as follows: nicotinic, salicylic, vanillic, gallic, syringic, $p$-coumaric, ferulic, sinapic, and caffeic acids. 
Sorbic and benzoic acids eluted at dead time. Nicotinic, salicylic, and vanillic acids eluted almost at the same time.

(1) Selection of the buffer $\mathrm{pH}$, concentration, and composition

Based on the preliminary studies of our systems by the CE method, we selected citrate $(\mathrm{CB})$ and phosphate (PB) buffers [18]. The $\mathrm{pH}$ of the buffers was maintained at 6.5. The concentration of the buffer solution was varied in the $15-35 \mathrm{mM}$ range with an organic solvent-methanol content of $10 \%$. This allowed us to obtain acceptable retention factors $k$ (1.01-3.11). There was not observed any significant effect of the buffer solution concentration on acid retention factors. Hence, for further experiments, a concentration of $15 \mathrm{mM}$ was chosen.

The $\mathrm{pH}$ of the buffer solution was varied in the 4.8-7.6 range. Mobile phase composition: $15 \mathrm{mM}$ buffer solution/methanol $(90: 10)$. For SP-1, a decrease in retention factors was observed for most acids with an increase in the $\mathrm{pH}$ of the buffer solution to 6.5. Sorbic, benzoic, and nicotinic acids were poorly retained on the sorbent in the entire studied $\mathrm{pH}$ range.

It is noteworthy that salicylic, gallic, and syringic acids behave differently from the general trend, as their retention factor varies little in the $\mathrm{pH}$ range of 4.8-5.6 (Figure 3). With a further increase in the $\mathrm{pH}$ of the buffer solution, their retention decreases as for all other acids.

The obtained dependencies are complex and are influenced both by the ionic state of acids and by the protonation degree of DS sulfo groups. The best selectivity with respect to the most PA was observed at $\mathrm{pH} 4.8$ (up to 1.52). For the separation of PA, PB with $\mathrm{pH} 4.8$ was chosen. To reduce the time for optimizing other chromatographic parameters, a citrate buffer solution was used.

The use of gradient elution regimes in subsequent studies will reduce the analysis time. To use the studied sorbents in HPLC-MS methods, it is important to use a volatile buffer to prevent the contamination of the ion source and the MS interface. We have already demonstrated earlier for similar sorbents [21] that solvent mixtures containing $\mathrm{CH}_{3} \mathrm{COOH} / \mathrm{CH}_{3} \mathrm{COONH}_{4}$ can be successfully used as such. Buffer capacity in this case plays a lesser role compared to the HPLC-UV since the amounts of the injected substances are significantly smaller.

(2) The choice of the organic modifier type and its content in the mobile phase

The influence of the nature of the organic modifier on acid retention was studied using acetonitrile, methanol, isopropanol, and THF (Table 1). In fact, only three of the solvents suitable for hydrophilic and reverse-phase HPLC-acetonitrile, methanol, and THF-have significantly different selectivity. Isopropanol was added to the study due to the widespread practice of its use in various HPLC modes. A water-organic mixture of the composition: $15 \mathrm{mM} \mathrm{CB}$ ( $\mathrm{pH}$ 6.5)/organic solvent (95:5) was used as a mobile phase. The acid elution order is the same for all four solvents in the composition of the mobile phase. Methanol has the least eluting power. Methanol is capable to discriminate all the analyzed PAs. Based on this result, methanol was selected for further work.

It is evident that acid retention is enhanced, and the eluting power of solvents decreases in the isopropanolacetonitrile-THF-methanol series. This series does not correlate with the order of the dielectric constant increase in the solvents. Usually, for the polar stationary phases, the eluting power of the solvent increases as its polarity grows, while for the nonpolar stationary phases, the reverse sequence is typical [22]. There is also a concept that the type of alcohol (and likely also other organic solvents) can influence the $3 \mathrm{D}$ structure of the polysaccharide-based stationary phases which ultimately leads to peculiar interactions with the analytes $[23,24]$. We cannot exclude this effect in our case. The resulting eluotropic series for SP-1 indicates its ambiguous mixed nature.

The influence of the methanol content in the mobile phase on chromatographic parameters was studied in the range from 0.5 to 90 vol. \% (Figure 4). A mixture of $15 \mathrm{mM}$ $\mathrm{CB}$ ( $\mathrm{pH}$ 6.5) and methanol was used as a mobile phase. The flow rate of the eluent was $1 \mathrm{ml} / \mathrm{min}$. The maximum retention for most acids was observed at a methanol content of $0.5 \%$ and the minimum at $90 \%$. For nicotinic, vanillic, salicylic, and gallic acids, the values of retention factors do not change in the 0.5 to $10 \%$ range but decrease in the range from 25 to $90 \%$. However, for ferulic and $p$-coumaric acids, a small minimum is observed at $70 \%$ (as U-shape retention profile for stationary phases carrying different interaction cites or as the dependence characteristic for HILIC hydrophilic chromatography).

Of the greatest interest to us were mobile phases with a methanol content of $0.5,5$, and $10 \%$, due to the strongest retention of most acids. The selectivity of their separation varies slightly in this interval. The maximum values of column efficiency (up to $16000 \mathrm{TP} / \mathrm{m}$ ) were observed at $10 \%$ methanol content; therefore, we chose it for the separation of the PA test mixture.

3.1.2. Silica Gel Twice Modified with 6,10-Ionene and Dextran Sulfate (SP-2). SP-2 contains 4 polyelectrolyte layers on its surface: 6,10-ionene-DS-6,10-ionene-DS. Under identical conditions, PA eluted from the surface of SP-2 slower than from the surface of SP-1. The procedure for eluting acids from the surface of SP-2 was as follows: nicotinic, sorbic, benzoic, salicylic, syringic, gallic, vanillic, sinapic, $p$-coumaric, ferulic, and caffeic. The sorbent was studied according to the same scheme as SP-1.

(1) Selection of the buffer $\mathrm{pH}$, concentration, and composition

With an increase in the concentration of $\mathrm{CB}$, the PA retention on SP-2 increases, but the selectivity of 


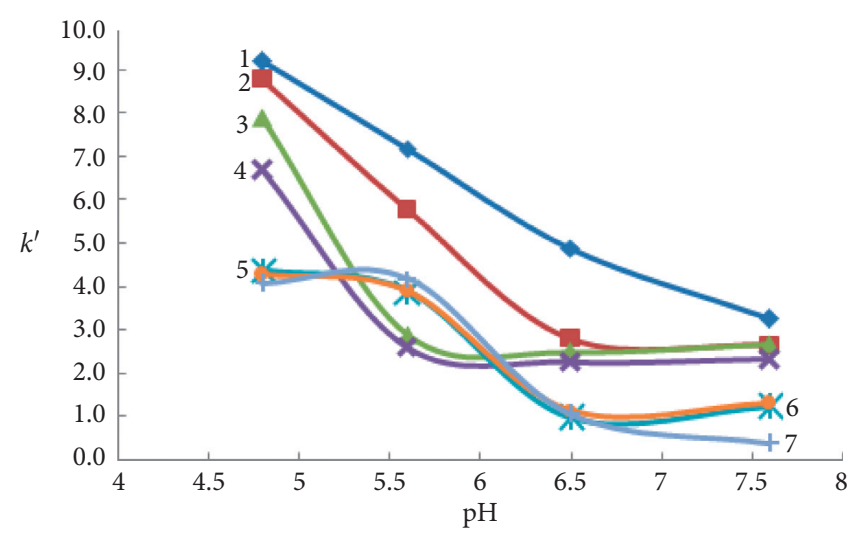

Figure 3: PA retention factors from buffer $\mathrm{pH}$ at SP-1 sorbent. Acids $(100 \mu \mathrm{g} / \mathrm{ml}): 1$, caffeic; 2 , sinapic; 3, ferulic; 4, $p$-coumaric; 5 , salicylic; 6 , syringic; 7 , gallic.

TABLE 1: Effect of the nature of the organic solvent in the mobile phase composition on the PA retention and the efficiency of the SP-1 column.

\begin{tabular}{|c|c|c|c|c|c|c|c|c|c|c|c|c|}
\hline \multirow{3}{*}{ Acid } & \multicolumn{12}{|c|}{ Organic solvent } \\
\hline & \multicolumn{3}{|c|}{ Acetonitrile } & \multicolumn{3}{|c|}{ Methanol } & \multicolumn{3}{|c|}{ THF } & \multicolumn{3}{|c|}{ Isopropanol } \\
\hline & $k^{\prime}$ & $N, \mathrm{TP} / \mathrm{m}$ & $\alpha$ & $k^{\prime}$ & $N, \mathrm{TP} / \mathrm{m}$ & $\alpha$ & $k^{\prime}$ & $N, \mathrm{TP} / \mathrm{m}$ & $\alpha$ & $k^{\prime}$ & $N, \mathrm{TP} / \mathrm{m}$ & $\alpha$ \\
\hline Caffeic & 0.82 & 2600 & 1.15 & 3.11 & 5900 & 1.07 & 0.93 & 2000 & 1.13 & 0.72 & 2300 & 1.16 \\
\hline Sinapic & 0.71 & 2500 & 1.01 & 2.92 & 7900 & 1.16 & 0.82 & 2000 & 1.11 & 0.62 & 2200 & 1.24 \\
\hline Ferulic & 0.70 & 1400 & 1.17 & 2.51 & 9700 & 1.14 & 0.74 & 2200 & 1.17 & 0.50 & 2200 & 1.00 \\
\hline$p$-Coumaric & 0.60 & 2900 & 2.50 & 2.20 & 9800 & 1.86 & 0.63 & 2300 & 2.03 & 0.50 & 2900 & 3.85 \\
\hline Gallic & 0.24 & 1400 & 1.14 & 1.18 & 7100 & 1.17 & 0.31 & 1900 & 1.00 & 0.13 & 1800 & 1.08 \\
\hline Vanillic & 0.21 & 800 & & 1.01 & 11600 & & 0.30 & 1900 & & 0.12 & 2100 & \\
\hline
\end{tabular}

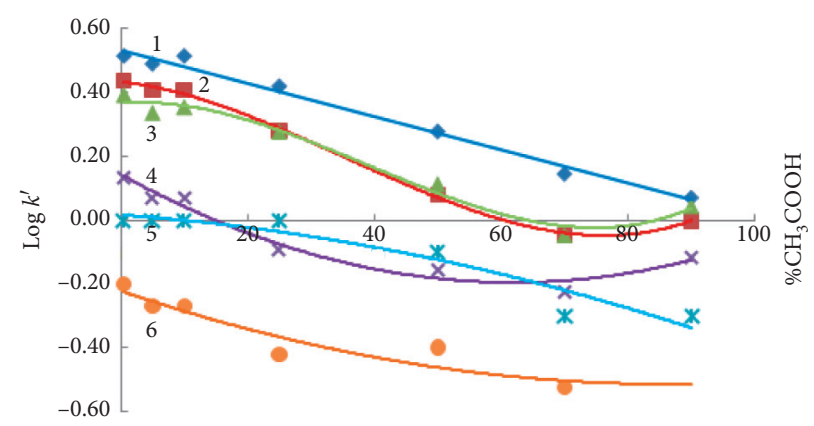

FIgURE 4: Effect of the methanol content in the mobile phase on the PA retention on SP-1. Acids (100 $\mu \mathrm{g} / \mathrm{ml}): 1$, caffeic; 2 , ferulic; 3 , p-coumaric; 4 , syringic; 5 , salicylic; 6 , nicotinic.

their separation remains practically unchanged. Consequently, the concentration of $15 \mathrm{mM}$ was selected for further experiments.

To assess the effect of $\mathrm{pH}$ on the retention of $\mathrm{PC}$, a mixture of acetonitrile and buffer solution was used as the mobile phase $(10: 90)$. With $\mathrm{pH}$ in 4.8-7.6 range, it was found that the retention factor for almost all compounds decreases with increasing $\mathrm{pH}$ (Figure 5). The effect is the most pronounced for caffeic, ferulic, p-coumaric, sinapic, vanillic, and salicylic acids. At pH 7.6, PA is already difficult to separate due to low selectivity. Hence, $\mathrm{PB}$ with $\mathrm{pH} 4.8$ was chosen for further work.
The pKa of the studied PA is in the 4.18-4.77 range (except for salicylic acid: 2.70). Thus, in aqueous solutions in the $4.8-7.6 \mathrm{pH}$ range, they are expected to be both in the dissociated and the protonated forms, and the total negative charge increases with increasing $\mathrm{pH}$. Besides, with an increase in $\mathrm{pH}$, the ionic state of the SD changes, and the fraction of the charged form increases. For SP-2, the maximum acid retention is observed at $\mathrm{pH} 4.8$ and the minimum at $\mathrm{pH}$ 7.6. This may be due to a decrease in hydrophobic and adsorption interactions and an increase in repulsive electrostatic interactions with increasing $\mathrm{pH}$. Thus, when the acids are more dissociated, their retention is weaker retained on this sorbent. 


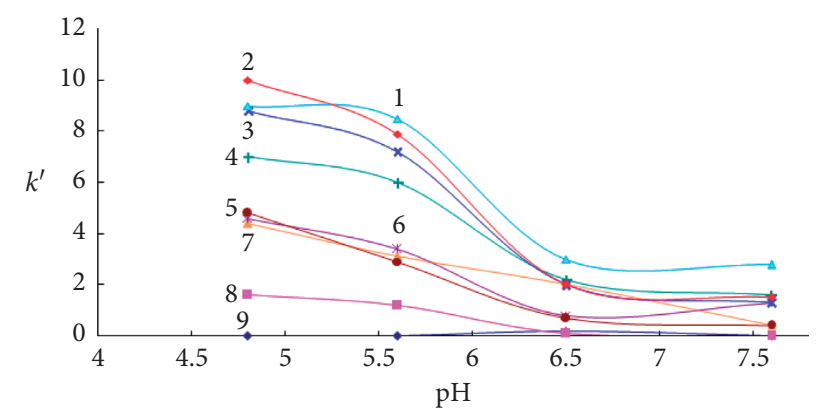

FIGURE 5: Effect of $\mathrm{pH}$ on the retention of PA on SP-2. Acids (100 $\mu \mathrm{g} / \mathrm{ml}): 1$, caffeic; 2 , ferulic; 3, p-coumaric; 4, sinapic; 5, vanillic; 6, gallic; 7 , salicylic; 8 , sorbic; 9 , benzoic.

(2) The choice of the organic modifier type and its content in the mobile phase

The influence of the nature of the solvent on acid retention was evaluated using the same solvents as in the case of SP-1. To reduce the time, we used a buffer solution with a relatively high $\mathrm{pH}$ of 6.5 , where the acid retention factor is below 4 (Table 2): mobile phase: organic modifier and $15 \mathrm{mM} \mathrm{CB}(\mathrm{pH}$ 6.5) (10:90). According to the obtained results, acetonitrile was chosen for further work as a comparison between satisfactory acid separation selectivity and column efficiency.

As evident from Table 2, the eluting strength of the solvents decreases in the isopropanol-acetonitrilemethanol-THF series. When using THF, a seemingly anomalous increase in acid retention is observed in comparison with all other solvents. This behavior is probably due to the ability of its molecules to form donor-type hydrogen bonds due to the presence of free electron pairs on the oxygen atom. In the case of SP-2, the surface of the sorbent is coated with an anionic polymer-DS, which does not have surface protons for interaction with THF. Thus, THF does not significantly affect the interaction of the sorbent with analytes, which leads to increased retention.

When assessing the effect of the acetonitrile content in the mobile phase on the behavior of PA, the content of the organic modifier was varied in the $0.5-70 \%$ range. It was found that an increase in acetonitrile content in the $0-50 \%$ range leads to a weakening of the acid retention, and with a further increase in acetonitrile content, acid retention factors increase (Figure 6). The highest selectivity for acid separation is observed with an acetonitrile content of $5 \%$.

3.1.3. Separation of Phenolic Acids on SP-1. Based on our studies, the following conditions were proposed for the separation of PA on SP-1: mobile phase composition $15 \mathrm{mM} \cdot \mathrm{PB} \quad(\mathrm{pH}$ 4.8)/methanol $(90: 10)$ and flow rate $F=0.5 \mathrm{ml} / \mathrm{min}$. A seven-component mixture of acids was separated (Figure 7). The column efficiency did not exceed
$6000 \mathrm{TP} / \mathrm{m}$; the separation selectivity was $1.04-1.65$, but the resolution between some peaks was less than $1.5(0.71-2.01)$.

3.1.4. Separation of Phenolic Acids on SP-2. The following chromatographic conditions were selected for the separation of PA on SP-2: mobile phase composition $15 \mathrm{mM} \cdot \mathrm{PB}(\mathrm{pH}$ 4.8)/acetonitrile (95:5) and flow rate $F=1 \mathrm{ml} / \mathrm{min}$. Under these conditions, a six-component mixture was separated in 15 minutes with the selectivity up to 1.88 and the column efficiency up to $26000 \mathrm{TP} / \mathrm{m}$ (Figure 8 ).

3.2. Separation of a Mixture of Phenolic Acids and NitrogenContaining Compounds on SP-2. We decided to evaluate the possibility of separating a mixture of basic and acidic compounds on the more successful sorbent SP-2 (Figure 9 and Table 3). When choosing the conditions for chromatography, we used the results obtained in this and previous works $[18,19]$. Separation on SP-2 is longer, but the selectivity and resolution of the peaks in the chromatogram are, in most cases, higher than when using SP-1. Thus, to determine a large number of components in various objects, especially with a complex matrix, it is better to use SP-2. The separation was carried out using a mixture of $15 \mathrm{mM}$ PBS (pH 5.6) and acetonitrile (95:5) as a mobile phase. The flow rate was $0.7 \mathrm{ml} / \mathrm{min}$.

3.3. Assumptions on the Mechanisms of Retention of Polar Phenolic Compounds on the Studied Stationary Phases. Phenolic acids used in this work contain groups that will provide dispersive (hydrophobic) interactions (the methylene group and the aromatic ring for example) with the surface of sorbents, induced dipole interactions (via aromatic ring), polar interactions through the carbonyl and hydroxyl groups, including the formation of hydrogen bonds, and also ion interaction through acid groups. The electron-donating methoxy and hydroxy groups in the acid composition also affect the interaction with the surface of the obtained stationary phases. Also, it is impossible to exclude the incomplete "closure" of the quaternary ammonium centers of 6,10-ionene on the surface of the sorbents during the deposition of subsequent layers of DS (especially in the case of SP-1). 
TABLE 2: Effect of the nature of the organic solvent in the mobile phase composition on the PA retention and the efficiency of the SP-2 column.

\begin{tabular}{|c|c|c|c|c|c|c|c|c|c|c|c|c|}
\hline \multirow{3}{*}{ Acid } & \multicolumn{12}{|c|}{ Organic solvent } \\
\hline & \multicolumn{3}{|c|}{ Acetonitrile } & \multicolumn{3}{|c|}{ Methanol } & \multicolumn{3}{|c|}{ THF } & \multicolumn{3}{|c|}{ Isopropanol } \\
\hline & $k^{\prime}$ & $N, \mathrm{TP} / \mathrm{m}$ & $\alpha$ & $k^{\prime}$ & $N, \mathrm{TP} / \mathrm{m}$ & $\alpha$ & $k^{\prime}$ & $N, \mathrm{TP} / \mathrm{m}$ & $\alpha^{*}$ & $k^{\prime}$ & $N, \mathrm{TP} / \mathrm{m}$ & $\alpha$ \\
\hline Caffeic & 2.59 & 16000 & 1.25 & 2.83 & 11000 & 1.10 & 3.92 & 10000 & 1.40 & 1.79 & 12000 & 1.05 \\
\hline Sinapic & 2.08 & 19000 & 1.09 & 2.58 & 9000 & 1.10 & 2.67 & 13000 & 1.02 & 1.70 & 13000 & 1.16 \\
\hline Ferulic & 1.90 & 14000 & 1.11 & 2.35 & 11000 & 1.69 & 2.81 & 12000 & 1.03 & 1.47 & 11000 & 1.07 \\
\hline$p$-Coumaric & 1.71 & 20000 & 2.41 & 1.39 & 23000 & 1.20 & 2.75 & 15000 & 2.38 & 1.37 & 21000 & 1.19 \\
\hline Gallic & 0.71 & 15000 & 1.18 & 1.16 & 11000 & 1.22 & 1.12 & 12000 & 1.23 & 1.15 & 11000 & 1.21 \\
\hline Vanillic & 0.60 & 17000 & & 0.95 & 15000 & & 0.91 & 18000 & & 0.95 & 15000 & \\
\hline
\end{tabular}

*The selectivity coefficient was calculated according to the elution order and not the location in the table.

TABLE 3: Chromatographic parameters of the separation of a mixture of FCC and nitrogen-containing compounds.

\begin{tabular}{|c|c|c|c|c|c|}
\hline Compound & $t_{R}, \min$ & $k^{\prime}$ & $\alpha$ & $R_{s}$ & $N, \mathrm{TP} / \mathrm{m}$ \\
\hline Nadolol & 2.57 & 0.12 & 2.75 & 1.60 & 54000 \\
\hline Tetrahydrozoline & 3.07 & 0.33 & 1.88 & 1.47 & 21000 \\
\hline Atenolol & 3.72 & 0.62 & 1.95 & 4.67 & 18000 \\
\hline Sorbic acid & 5.07 & 1.21 & 1.17 & 1.43 & 33000 \\
\hline Benzoic acid & 5.54 & 1.41 & 1.28 & 2.01 & 28000 \\
\hline Carbinoxamine & 6.47 & 1.81 & 1.38 & 1.82 & 14000 \\
\hline Chlorpheniramine & 8.04 & 2.49 & 1.29 & 1.91 & 9000 \\
\hline Vanillic acid & 9.70 & 3.22 & 1.12 & 1.17 & 28000 \\
\hline Gallic acid & 10.63 & 3.62 & 2.44 & 9.00 & 12000 \\
\hline Sinapic acid & 22.63 & 8.84 & 1.31 & 3.18 & 20000 \\
\hline Caffeic acid & 29.00 & 11.61 & & & 16000 \\
\hline
\end{tabular}

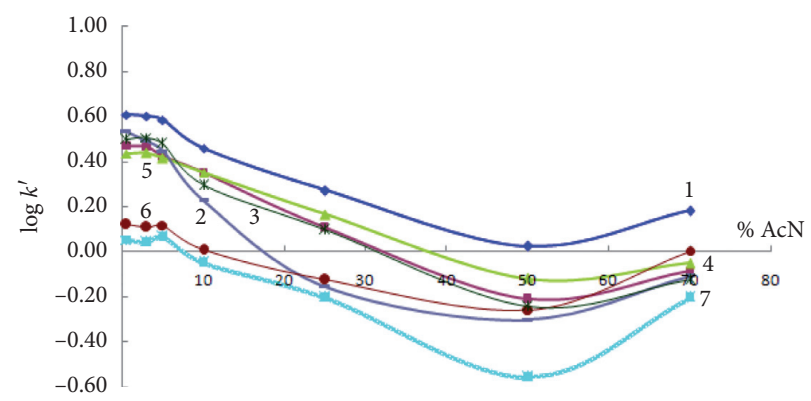

Figure 6: Effect of the acetonitrile content in the mobile phase on the PA retention on SP-2. Acids (100 $\mu$ g/ml): 1, caffeic; 2, sinapic; 3 , salicylic; 4, ferulic; 5, p-coumaric; 6 , gallic; 7, vanillic.

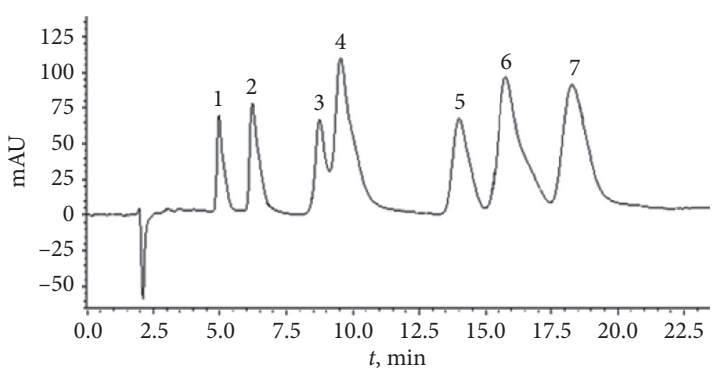

FIgURe 7: Chromatogram of a mixture of PA on a column with sorbent-4. Acids $(15 \mu \mathrm{g} / \mathrm{ml})$ : 1 , sorbic; 2 , nicotinic; 3 , vanillic; 4 , gallic; 5 , p-coumaric; 6, ferulic; 7, caffeic. 


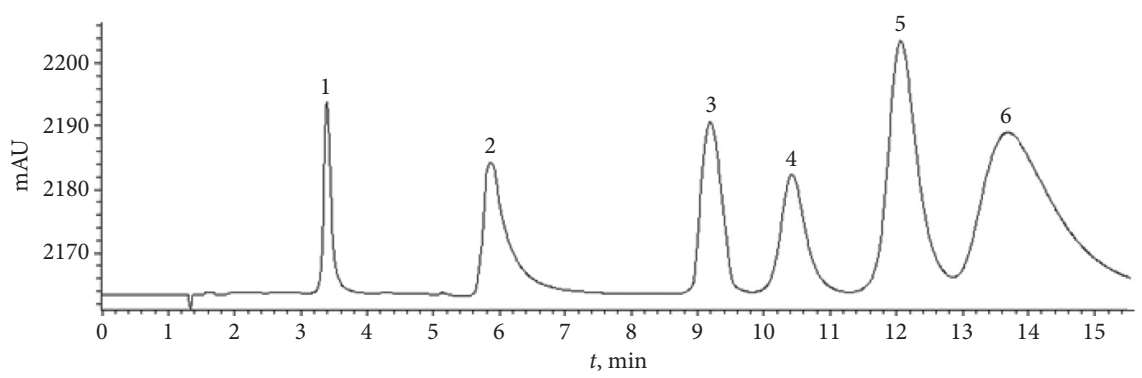

FIGURE 8: Separation of PA on SP-2 column $(80 \times 4.6 \mathrm{~mm})$. Acids $(15 \mu \mathrm{g} / \mathrm{ml}): 1$, sorbic; 2, vanillic; 3, sinapic; 4, p-coumaric; 5, caffeic; 6 , ferulic.

TABLE 4: Hansch hydrophobicity parameters and PA retention factors on SP-1.

\begin{tabular}{lcc}
\hline Acid & $\log k^{\prime}$ & $\log P$ \\
\hline Nicotinic & -0.27 & 0.15 \\
Gallic & 0.04 & 0.91 \\
Syringic & 0.07 & 1.13 \\
Sinapic & 0.45 & 1.29 \\
Vanillic & 0 & 1.33 \\
Sorbic & - & 1.35 \\
Caffeic & 0.51 & 1.42 \\
Ferulic & 0.40 & 1.64 \\
p-Coumaric & 0.36 & 1.88 \\
Benzoic & - & 1.89 \\
Salicylic & 0 & 2.06 \\
\hline
\end{tabular}

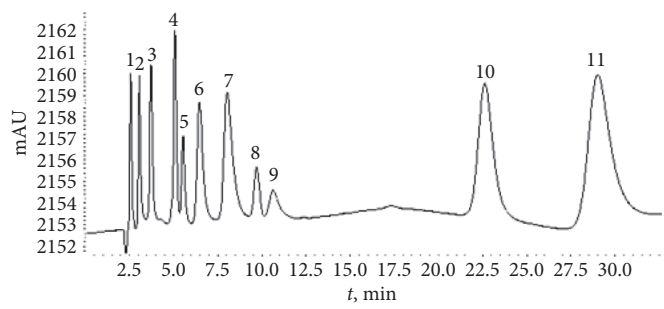

Figure 9: Separation of PA and nitrogen-containing compounds on the SP-2 column $(150 \times 4.6 \mathrm{~mm})$. Compounds $(0.32-15 \mu \mathrm{g} / \mathrm{ml})$ : 1, nadolol; 2, tetrahydrozoline; 3 , atenolol; 4 , sorbic acid; 5, benzoic acid; 6 , carbinoxamine; 7, chlorpheniramine; 8 , vanillic acid; 9 , gallic acid; 10, sinapic acid; 11, caffeic acid.

Several possible options for the acid-sorbent interactions make studying the nature of the sorbents quite difficult. Therefore, we can make some of the clearest, in our opinion, assumptions about the PA retention mechanisms on the obtained stationary phases.

\subsubsection{Silica Gel Modified with 6,10-Ionene and Dextran} Sulfate (SP-1). Analyzing the results of the PA retention study SP-1 (silica gel, modified with 6,10-ionene and dextran sulfate), its ambiguous nature can be noted.

For example, the contribution of hydrophobic interactions to the retention mechanism is confirmed by the fact that acid retention is noticeably weakened with an increase in the methanol content in the mobile phase (Figure 4). However, the dependence of the PA retention on the organic solvent content on this sorbent does not have the form, characteristic of reverse-phase chromatography for all acids. It seems that hydrophilic interactions sorbate-sorbent also makes a significant contribution to the retention mechanism. This is evidenced by the ambiguous nature of the influence of the organic solvent on the retention of substances.

The $\mathrm{pKa}$ of the studied PAs is in the range of 4.18-4.77 (except 2.70 for salicylic acid). Thus, in aqueous solutions in the 4.8-7.6 $\mathrm{pH}$ range, they are found both in the undissociated and anionic forms, and with an increase in $\mathrm{pH}$, the number of 
anions in the solution increases. Besides, with an increase in $\mathrm{pH}$, the ionic state of DS changes, and the fraction of the charged form (DS-polyanion) increases. For SP-1, the maximum acid retention is observed at $\mathrm{pH} 4.8$ and the minimum at $\mathrm{pH}$ 7.6. This may be due to the weakening of the hydrophobic and the adsorption sorbate-sorbent interactions and the strengthening of the repulsive electrostatic interactions with increasing $\mathrm{pH}$. This assumption is confirmed by the fact that salicylic acid (completely dissociated under experimental conditions) is not retained on the sorbent. Noteworthy, nicotinic acid, due to the presence of the main center in the pyridine ring, is retained on the sorbent stronger than benzoic acid. At low $\mathrm{pH}$, this pyridine ring will be present in solution predominantly in the protonated form. This may lead to stronger interactions with sulfate groups on the dextran sulfate surface.

In confirmation of the mixed hydrophilic-hydrophobic nature of the SP-1 sorbent, we compared the elution order of PA with their Hansch hydrophobicity parameters, which are shown in Table 4 . The acids are arranged by their hydrophilicity in descending order. These data show that, for them, there is no definite relationship between the PA retention and $\log P$.

The retention time increases in the series of salicylic $<p$ coumaric $<$ ferulic < caffeic acids; in the same series, the Hansch hydrophobicity parameter decreases, and the hydrophilicity of the compounds increases (Table 4). But retention on the sorbent is also enhanced in the series of nicotinic $<$ gallic $<$ syringic $<$ sinapic $<$ caffeic acids, which correlate well with an increase in their hydrophobicity. All the obtained patterns confirm the mixed hydrophilic-hydrophobic nature of the synthesized sorbent.

According to the data from [25], similar patterns were also noted for nitrogen-containing compounds. The compounds considered were pindolol (1.97), nadolol (1.29), atenolol (0.10), chlorpheniramine (3.39), carbinoxamine (2.76), hydroxyzine (2.03), doxylamine (4.01), terbutaline (0.48), and tetrahydrozoline (3.31) (Figure 10). Their Hansch hydrophobicity parameters are given in parenthesis. The dependence of their retention on the electrolyte concentration is similar to that for PA. The effect of the organic solvent content on the retention of nitrogen-containing compounds is better described by the laws of hydrophilic chromatography than in the PA case. The U-shaped dependence of the retention of the studied nitrogen-containing pharmaceuticals on the methanol content in the mobile phase additionally confirms it.

A definite relationship between the hydrophobicity parameter and the retention of nitrogen-containing bases was not observed for all the studied compounds. However, in general, with an increase in hydrophobicity, nitrogencontaining compounds are retained weaker on SP-1 (Table 5). Thus, with the complex nature of SP-1, the retention of polar compounds is described mainly by the laws of hydrophilic chromatography.
3.3.2. Silica Gel Twice Modified with 6,10-Ionene and Dextran Sulfate (SP-2). The nature of the PA retention dependence on the organic solvent content on SP-2 corresponds to the laws of reversed-phase chromatography. However, the influence of the organic mobile phase modifier on acid retention factors has a less pronounced dependence.

To assess the contribution of hydrophobic and adsorption interactions to the retention mechanism, we also compared the retention factors of the studied compounds with their Hansch hydrophobicity parameter (Table 6). The acids in the table are arranged by their retention factors in ascending order. It is evident that not always an increase in hydrophobicity leads to increased retention on SP-2. But such a relationship can still be noticed if we select molecules similar in structure. In the series nicotinic-sorbic-benzoicsalicylic acids, the retention factor increases with increasing $\log P$ parameter. Vanillic acid is retained stronger than syringic and caffeic, which in turn are retained longer than synaptic, and this pattern correlates with an increase in their $\log P$.

Sinapic, $p$-coumaric, ferulic, and caffeic acids, in the structure of which there is a 2-carboxyethyl aryl fragment (Figure 11), were retained stronger on SP-2 than other acids.

The elution order of these acids clearly depends on the number and nature of the substituents in the benzene ring. The maximum retention factor was observed for caffeic acid with two $\mathrm{OH}$ groups, while for $p$-coumaric acid with one $\mathrm{OH}$ group and ferulic acid, where the $-\mathrm{OSH}_{3}$ group is additionally located in the o-position, it was somewhat smaller. A more noticeable decrease in retention was observed for sinapic acid where hydroxyl is shielded by two $-\mathrm{OSCH}_{3}$ groups. Likely, the retention of these acids on SP-2 is significantly determined by dipoledipole sorbate-sorbent interactions due to the formation of hydrogen bonds with the molecules of the DS modifier on the surface, where the influence of residual amino groups of 6,10-ionene and silanol groups of silica gel is likely less than observed for SP-1 and therefore can be neglected.

The retention of nitrogen-containing compounds [25] on SP-2 increases with their hydrophobicity (Figure 12), which means that, upon separation of nitrogen-containing compounds, the contribution of hydrophobic interactions to the retention mechanism increases. The contribution of dipole-dipole interactions also plays an important role since substances with a structure that contains either two benzene rings or an indole ring are more strongly retained on the sorbent.

Thus, SP-2 seems to be a mixed-mode sorbent, where hydrophobic and polar sorbate-sorbent interactions make a significant contribution to the retention mechanism. 
<smiles>CC(C)NCC(O)COc1cccc2[nH]ccc12</smiles><smiles>CC(C)(C)NCC(O)COc1cccc2c1C[C@H](O)[C@H](O)C2</smiles><smiles>CC(C)NCC(O)COc1ccc(CC(N)=O)cc1</smiles>

Atenolol<smiles>CN(C)CCOC(c1ccc(Cl)cc1)c1ccccn1</smiles>

Carbinoxamine<smiles>OCCOCCN1CCN(C(c2ccccc2)c2ccc(Cl)cc2)CC1</smiles>

Hydroxyzine

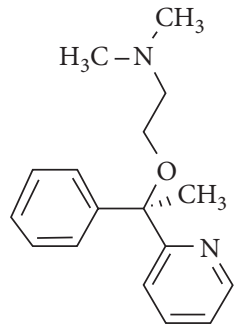

Doxylamine<smiles>CC(C)(C)NCC(O)c1cc(O)cc(O)c1</smiles>

Terbutaline<smiles>c1ccc2c(c1)CCCC2C1=NCCN1</smiles>

Tetrahydrozoline

Figure 10: Chemical structure of basic nitrogen-containing compounds.

TABLE 5: Hansch hydrophobicity parameters and nitrogen-containing compounds retention factors on SP-1.

\begin{tabular}{lcc}
\hline Base & $\log k^{\prime}$ & $\log P$ \\
\hline Chlorpheniramine & -0.52 & 3.39 \\
Carbinoxamine & -0.40 & 2.76 \\
Nadolol & -0.10 & 1.29 \\
Tetrahydrozoline & 0.28 & 3.31 \\
Pindolol & 0.49 & 1.97 \\
\hline
\end{tabular}

TABLE 6: Hansch hydrophobicity parameters and PA retention factors on SP-2.

\begin{tabular}{lcc}
\hline Acid & $\log k^{\prime}$ & $\log P$ \\
\hline Nicotinic & 0.03 & 0.15 \\
Sorbic & 0.20 & 1.35 \\
Benzoic & 0.23 & 1.89 \\
Salicylic & 0.64 & 2.06 \\
Syringic & 0.65 & 1.13 \\
Gallic & 0.66 & 0.91 \\
Vanillic & 0.68 & 1.33 \\
Sinapic & 0.85 & 1.29 \\
p-Coumaric & 0.94 & 1.88 \\
Ferulic & 0.95 & 1.64 \\
Caffeic & 1.06 & 1.42 \\
\hline
\end{tabular}


<smiles>[R]c1cc(/C=C/C(=O)O)cc([R])c1[X]</smiles>

FIGURE 11: 2-Carboxyethyl aryl fragment.

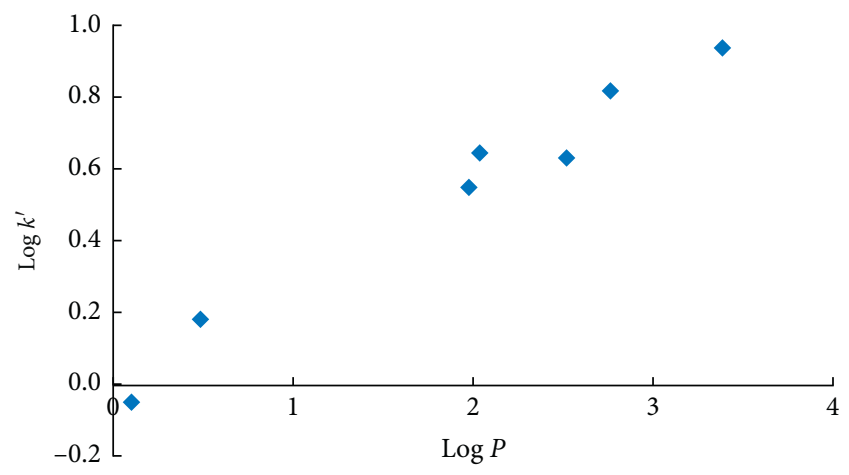

Figure 12: Relationship between the retention factors of nitrogencontaining compounds on SP-2 and their hydrophobicity parameters.

\section{Conclusions}

Summarizing the data obtained in this work and previous studies, the following can be concluded. With the ambiguous retention mechanism of polar acids and bases on SP-1, it can be assumed that the separation of this class of compounds proceeds predominantly by the hydrophilic chromatography mechanism. SP-2, in turn, can be considered a mix-mode stationary phase.

The retention of polar phenolic acids, the selectivity of their separation, together with the column efficiency increases with the transition from SP-1 to SP-2. Thus, it follows the general trend that an increase in the number of layers in a multilayer coating improves the parameters of the separation systems [16, 17, 26, 27].

The possibility of separating the two classes of compounds-phenolic acids and basic nitrogen-containing pharmaceuticals -in a single mixture on silica gel, twice modified with 6.10-ionene and dextran sulfate, is shown. It is possible to expand the list of polar analytes in the future. Synthesized stationary phases can be recommended for the separation and analysis of the different polar compounds in the pharmaceutical, food, and other industries.

\section{Data Availability}

The data used to support the findings of this study are included within the article.

\section{Conflicts of Interest}

The author declares that there are no conflicts of interest.

\section{References}

[1] W. Zhou, S. Liu, W. Ju et al., "Simultaneous determination of phenolic acids by UPLC-MS/MS in rat plasma and its application in pharmacokinetic study after oral administration of Flos Lonicerae preparations," Journal of Pharmaceutical and Biomedical Analysis, vol. 86, pp. 189-197, 2013.

[2] I. Ali, U. Kulsum, Z. A. AL-Othman, and K. Saleem, "Analyses of nonsteroidal anti-inflammatory drugs in human plasma using dispersive nano solid-phase extraction and high-performance liquid chromatography," Chromatographia, vol. 79, no. 3-4, pp. 145-157, 2016.

[3] E. Porgali and E. Buyuktuncel, "Determination of phenolic composition and antioxidant capacity of native red wines by high performance liquid chromatography and spectrophotometric methods," Food Research International, vol. 45, pp. 145-154, 2012.

[4] M. M. P. Natividade, L. C. Corrêa, S. V. C. d. Souza, G. E. Pereira, and L. C. d. O. Lima, "Simultaneous analysis of 25 phenolic compounds in grape juice for HPLC: method validation and characterization of São Francisco Valley samples," Microchemical Journal, vol. 110, pp. 665-674, 2013.

[5] I. Ginjom, B. D’Arcy, N. Caffin, and M. Gidley, "Phenolic compound profiles in selected Queensland red wines at all stages of the wine-making process," Food Chemistry, vol. 125, no. 3, pp. 823-834, 2011.

[6] M. R. Siddiqui, Z. A. AlOthman, and N. Rahman, "Analytical techniques in pharmaceutical analysis: a review," Arabian Journal of Chemistry, vol. 10, pp. S1409-S1421, 2017.

[7] K. Kardani, N. Gurav, B. Solanki, P. H. Patel, and B. C. Patel, "RP-HPLC method development and validation of gallic acid in polyherbal tablet formulation," Journal of Applied Pharmaceutical Science, vol. 3, no. 5, pp. 37-42, 2013.

[8] N. Hasan, M. Chaiharn, U. A. Toor et al., "Development, validation and application of RP-HPLC method: simultaneous determination of antihistamine and preservatives with paracetamol in liquid formulations and human serum," The Open Medicinal Chemistry Journal, vol. 10, no. 1, pp. 33-43, 2016.

[9] A. J. Alpert, M. Shukla, A. K. Shukla et al., "Hydrophilicinteraction chromatography of complex carbohydrates," Journal of Chromatography A, vol. 676, no. 1, pp. 191-202, 1994.

[10] P. Jandera, "Stationary and mobile phases in hydrophilic interaction chromatography: a review," Analytica Chimica Acta, vol. 692, no. 1-2, pp. 1-25, 2011.

[11] A. Shen, Z. Guo, and X. Liang, "Development and application of hydrophilic interaction liquid chromatographic stationary phases," Progress in Chemistry, vol. 26, pp. 10-18, 2014.

[12] M. Liu, E. X. Chen, R. Ji, and D. Semin, "Stability-indicating hydrophilic interaction liquid chromatography method for highly polar and basic compounds," Journal of Chromatography A, vol. 1188, no. 2, pp. 255-263, 2008.

[13] J. Shen, T. Ikai, and Y. Okamoto, "Synthesis and application of immobilized polysaccharide-based chiral stationary phases for enantioseparation by high-performance liquid chromatography," Journal of Chromatography A, vol. 1363, pp. 51-61, 2014.

[14] N. L. T. Padivitage and D. W. Armstrong, "Sulfonated cyclofructan 6 based stationary phase for hydrophilic interaction chromatography," Journal of Separation Science, vol. 34, no. 14, pp. 1636-1647, 2011.

[15] P. Ding, B. Chang, G. Qing, and T. Sun, "New approach for chiral separation: from polysaccharide-based materials to 
chirality-responsive polymers," Science China-Chemistry, vol. 57, pp. 1492-1506, 2014.

[16] C. Liu and J. Kang, "Improved capillary electrophoresis frontal analysis by dynamically coating the capillary with polyelectrolyte multilayers," Journal of Chromatography A, vol. 1238, pp. 146-151, 2012.

[17] Z. D. Kihm, E. M. Veen, J. D. Bergen-Hartigan, Y. Zhang, and Y. Liu, "Modification of electroosmotic flow for a polydimethylsiloxane electrophoresis microchip via polyelectrolyte coating," Analytical Sciences, vol. 28, no. 2, pp. 183-186, 2012.

[18] A. Ioutsi, E. Shapovalova, A. Prokhorova, and O. Shpigun, "Layer-by-layer assembly of polysaccharides and 6, 10-ionene for separation of nitrogen-containing pharmaceuticals and their enantiorecognition by capillary electrophoresis," Journal of Chemistry, vol. 2015, Article ID 836076, 2015.

[19] A. N. Ioutsi, E. N. Shapovalova, V. A. Ioutsi, A. G. Majouga, and O. A. Shpigun, "Separation of nitrogen containing pharmaceuticals on silica gels modified with 6.10-ionene, dextran sulfate and gold," Journal of Physical Chemistry, vol. 91, no. 12, p. 2115, 2017 in Russian.

[20] J. L. -M. Abboud, R. Notario, J. Bertran, and M. Sola, Progress in Physical Organic Chemistry, Willey Online Library, Hoboken, NJ, USA, 1993.

[21] A. N. Ioutsi, V. A. Ioutsi, E. N. Shapovalova, and O. A. Shpigun, "Determination of pharmacologically active nitrogen-containing compounds on silica doubly modified with 6,10-Ionene and dextran sulphate by high-performance liquid chromatography-tandem mass spectrometry," Journal of Analytical Chemistry, vol. 75, no. 7, pp. 930-935, 2020.

[22] B. A. Rudenko and G. I. Rudenko, High Efficiently Chromatographic Processes. Condensed Mobile Phases, Nature, Moscow, Russia, in Russian, 2003.

[23] L. Fumagalli, L. Pucciarini, L. Regazzoni et al., "Direct HPLC separation of carnosine enantiomers with two chiral stationary phases based on penicillamine and teicoplanin derivatives," Journal of Separation Science, vol. 41, no. 6, pp. 1240-1246, 2018.

[24] I. Ilisz, R. Berkecz, and A. Péter, "Retention mechanism of high-performance liquid chromatographic enantioseparation on macrocyclic glycopeptide-based chiral stationary phases," Journal of Chromatography A, vol. 1216, no. 10, pp. 18451860, 2009.

[25] A. N. Ioutsi, "Electophoretic and HPLC separation of polar compounds by materials layer-by-layer modified with polycations and polyanions," Ph. D. Dissertation, Lomonosov Moscow State University, Moscow, Russia, 2015, in Russian.

[26] J. Znaleziona, J. Petr, R. Knob, V. Maier, and J. Svĕcĭk, "Dynamic coating agents in CE," Chromatographia, vol. 67, pp. S5-S12, 2008.

[27] H. Katayama, Y. Ishihama, and N. Asakawa, "Stable capillary coating with successive multiple ionic polymer layers," $A n$ alytical Chemistry, vol. 70, no. 11, pp. 2254-2260, 1998. 